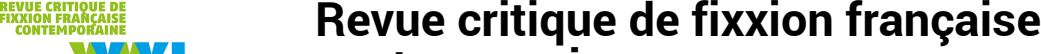 \\ XXI contemporaine
}

$22 \mid 2021$

Figures du mensonge et de la mauvaise foi dans le roman contemporain

\section{Mensonge et fiction : Paris-Brest et Article 353 du code pénal de Tanguy Viel}

\section{Claude Coste}

\section{OpenEdition}

\section{Journals}

Édition électronique

URL : https://journals.openedition.org/fixxion/337

DOI : $10.4000 /$ fixxion.337

ISSN : 2295-9106

Éditeur

Ghent University

Référence électronique

Claude Coste, « Mensonge et fiction : Paris-Brest et Article 353 du code pénal de Tanguy Viel », Revue critique de fixxion française contemporaine [En ligne], 22 | 2021, mis en ligne le 15 juin 2021, consulté le 17 février 2022. URL : http://journals.openedition.org/fixxion/337 ; DOI : https://doi.org/10.4000/ fixxion.337

Ce document a été généré automatiquement le 17 février 2022.

\section{(c) (i) $\odot$}

Les contenus de la Revue critique de fixxion française contemporaine sont mis à disposition selon les termes de la licence Creative Commons Attribution - Pas d'Utilisation Commerciale - Pas de Modification 4.0 International. 


\title{
Mensonge et fiction : Paris-Brest et Article 353 du code pénal de Tanguy Viel $^{1}$
}

\author{
Claude Coste
}

1 Quand il envisage d'écrire son "roman familial", Louis, le narrateur de Paris-Brest, hésite entre deux traditions littéraires : "D'un côté je voulais faire un roman familial à la française, de l'autre je voulais faire un roman à l'anglaise, et cela d'autant plus que tout se passe en Bretagne et pire qu'en Bretagne, dans le Finistère Nord, c'est-à-dire dans la partie la plus hostile, la plus sauvage et la plus rocheuse de Bretagne, alors c'était d'autant plus normal de donner à tout ça un côté, disons, irlandais, un côté Cornouailles avec des oiseaux noirs et des pierres fatiguées" (PB 73). On pourrait tout aussi bien imaginer une troisième tentation, tant la littérature et le cinéma américains jouent un rôle important dans l'œuvre de Tanguy Viel. Mais, malgré la présence de nombreuses références au monde anglo-saxon, Paris-Brest et Article 353 du code pénal sont, incontestablement, deux romans bien français et, de l'aveu même du narrateur, deux romans bretons, qui se déroulent à Brest ou dans sa périphérie. Dans un roman familial à la française, les questions d'argent sont essentielles, provoquant les guerres qui déchirent frères et sœurs, parents et enfants, individu et société, classe dominante et classes dominées.

Dans Paris-Brest, la grand-mère du narrateur épouse Albert dont elle accompagne les dernières années: elle hérite de dix-huit millions qui suscitent immédiatement la convoitise de sa fille, de son gendre et même de Louis, son petit-fils. Soupçonné de détournement de fonds, le père de Louis, accompagné de son épouse, s'installe quelques années à Palavas-les-Flots pour échapper à l'opinion publique. Refusant de suivre ses parents avec lesquels il entretient des relations tendues, Louis s'installe au rez-dechaussée de l'immeuble habité par sa grand-mère, avec laquelle il déjeune tous les dimanches au Cercle marin. Poussé par le "fils Kermeur", son mauvais génie, il organise le cambriolage de la vieille dame, sans éveiller les soupçons, ni de la police, ni de sa famille. Quand ses parents décident de revenir en Bretagne, d'acheter une maison en 
bord de mer, le jeune homme part à Paris où il écrit son roman. De retour à Brest pour Noël, il est l'objet de toutes les sollicitudes de la part des membres de sa famille qui craignent qu'une telle publication ne leur porte préjudice. Après une dernière crise, le narrateur repart pour Paris.

Dans Article 353 du code pénal, le titre, mystérieux, ne prendra sens qu'à la fin, après l'enquête menée par le juge qui interroge Martial Kermeur (le même nom que l'acolyte du narrateur de Paris-Brest) accusé de meurtre. Suite à une escroquerie immobilière qui dépouille de leurs indemnités les travailleurs de l'Arsenal, provoque le suicide de Le Goff, le maire de la commune, pousse Erwan Kermeur au vandalisme (le jeune homme saborde les bateaux amarrés dans le port de plaisance), Martial Kermeur, le narrateur, jette à l'eau le promoteur Antoine Lazenec, avec lequel il s'était jusqu'alors montré très complaisant. Racontant son histoire, Kermeur suscite progressivement la sympathie du juge qui conclut à un accident, arguant de l'article $353 \mathrm{du}^{\text {code pénal }}{ }^{2}$. Une fois encore, l'argent, l'appât du gain, les relations familiales difficiles (entre Kermeur et sa femme, entre Kermeur et son fils) construisent un univers à la fois breton et français, sur fond de crise économique et sociale.

Moins marqués par le monde anglo-saxon, les deux romans où règnent le mensonge et la mauvaise foi, où s'impose plus ou moins discrètement une réflexion méta-poétique sur la fiction narrative (Louis écrit un roman, le juge sonde les âmes et les cœurs comme un bon écrivain), dessinent un univers désenchanté dans lequel les personnages recherchent un hypothétique bonheur. Mais si l'on se propose de lire ces deux œuvres ensemble, ce n'est pas seulement pour une même approche qu'on qualifiera de "postmoderne", c'est aussi - et surtout - parce que Paris-Brest et Article 353 du code pénal placent les mots au premier plan de la scène romanesque.

\section{Mots, coups de fouet}

5 Peu de romans usent autant de l'autonymie. Si la fonction référentielle est omniprésente (comment faire autrement?), les personnages et les narrateurs ne cessent de désigner les mots comme mots, de signaler par l'emploi régulier des guillemets que tel ou tel signe est employé comme signe : "nous avons inventé un joli mot, et que ce joli mot, c'est 'espoir'. J'ai dit cela au juge, précisément cela, avec ce tonlà, pour que dans ma voix il puisse voir lui aussi les guillemets qui faisaient comme un liséré d'or autour du mot 'espoir"' (A 141). En poétisant la ponctuation, Martial Kermeur esthétise une pratique récurrente chez les personnages de Tanguy Viel.

Certes, la réalité et la communication ne se résument pas à une logosphère généralisée. Le jeune Erwan accorde une grande place aux images, iconographiques ou mentales ${ }^{3}$, comme moyen d'entrer en relation avec les autres; il manifeste, selon son père, une acuité du regard qui échappe à l'empire et à l'emprise des mots :

Je sais qu'il n'a pas eu besoin de savoir lire sur les lèvres pour établir l'état des forces en présence entre Lazenec et moi pour les subir, vous comprenez, parce que ces choses-là, il faudrait être vraiment naiff pour croire qu'elles se tiennent dans une langue faite de phrases, quand n'importe quel enfant de cinq ans sait déjà lire dans la courbure des épaules ou les mouvements de la nuque, sait déjà lire qui tient

l'autre et l'écrase d'une seule main. (A 143-144)

Dans Paris-Brest, l'homosexualité du frère de Louis n'a pas besoin d'être verbalisée pour devenir une évidence ("Des choses comme ça, j'ai dit, tout le monde le sait sans le dire" (PB 164)). Souvent superflus, les mots sont frappés de vanité quand ils tournent à vide ; 
c'est, grâce à une étrange métaphore, le cas des "petits agents commerciaux qu'on paye à la commission et dont toutes les phrases ont l'air d'être comme des coquillages collés sur le dos d'une baleine" (A 63) ; c'est le cas des conversations qui cherchent à mettre fin au malaise en misant sur "pire que le silence, les phrases pour l'effacer" (PB 156).

Malgré les non-dits ou la vacuité du dire, les mots se situent au cœur des aventures humaines, comme si Tanguy Viel et ses personnages voulaient restaurer le Livre du monde : "je vois se dessiner la ville, et elle me semble écrite dans une langue que je ne comprends pas, un alphabet fait d'immeubles reconstruits et de fenêtres ouvertes et seulement sur les rebords je peux repérer les miettes qui restent" (A 11). Dans ce monde hanté par les mots, les personnages investissent les phrases d'une dimension identitaire. Cela vaut pour le fils Kermeur (“Toujours garder en réserve de l'inadaptation', disait-il. C'était sa grande phrase" (PB 79); “Ta mère a avalé un cimetière" ( $P B$ 94)) ou pour son ennemie, la mère de Louis, qui juge ainsi son fils : "Je n'étais pas encore sec derrière les oreilles" ( $P B$ 98). Grâce à ces phrases-refrains, les personnages tentent de faire face au réel, comptant sur la dimension performative du langage, même si le vocabulaire de la linguistique est bien loin de leur univers. Qu'elle soit prise au sens strict ("quand dire, c'est faire") ou dans un sens élargi, la performativité correspond à une crainte et à un espoir. Dans le monde de Tanguy Viel, qu'il s'agisse de la vie ou de la création, les mots ne sont jamais innocents. Au sortir d'un siècle de misologie affectée, le romancier et ses personnages savent bien que les mots ne sont ni suffisants, ni insuffisants en soi. Ce qui importe, c'est l'utilisateur et sa capacité à faire entendre sa parole au sein de la relation sociale. Dotés d'une puissance évocatrice ("Dès qu'il a prononcé l'expression 'assiette de Limoges', j'ai vu la scène" ( $P B$ 150)), sources d'un plaisir parfois mauvais ("plaisir d'entendre dire du mal des gens qu'on connaît" (PB 35) ; "le bien que ça fait de dire du mal de son fils" (A 154)), les mots et les formules habitent les personnages qui les ressassent pour le meilleur et pour le pire $^{4}$. Les deux romans ne cessent de multiplier cet usage strict de la performativité qui sépare un avant et un après de la profération; on signe beaucoup dans Article 353 et Paris-Brest : un chèque (achat immobilier), un bail (l'appartement de Louis près du Luxembourg), un contrat (le mariage d'Albert et de la grand-mère) et la signature engage définitivement celui qui a franchi ce Rubicon d'encre et de papier ${ }^{5}$.

De manière générale, les mots font de l'effet. Ainsi une remarque de Le Goff ("faire une croix dessus") bouleverse Martial Kermeur :

Cette phrase-là, monsieur le juge, faire une croix dessus, monsieur le juge, je ne sais pas si j'ai compris tout ce qu'elle signifiait mais je sais que dans ma tête ça a fait comme une énorme bâche qui s'est mise à recouvrir la presqu'île tout entière, quelque chose comme une marée noire qui serait venue du fond de l'océan et aurait tapissé la rade de sa poisse (A 120)

Quant à la dernière partie de Paris-Brest, elle est entièrement organisée autour d'une phrase lancinante qui circule d'un membre à l'autre de la famille, tous inquiets que Louis dans son "roman familial" n'ait écrit : "Des choses sur nous".

9 Retrouvant Eschyle, Tanguy Viel compare le mot à un "fouet dans l'air"' (A 26), et les phrases à des "flèches" (A 33) S'ils bénéficient d'une telle puissance, c'est que les mots ont partie liée à l'argent. Contrairement au monde de Gide, les faiseurs de phrases chez Tanguy Viel ne sont pas des faux-monnayeurs par nature. Entre les mots et l'argent se met en place une étrange dialectique, comme si les deux partenaires se nourrissaient l'un de l'autre. L'argent existe sans les mots et les mots sans l'argent; mais ils ne tardent pas à entrer dans une émulation ou une complicité quasi diabolique. Relayé par 
les mots ("propriétaire" ( $P B$ 32), "héritière", "six-huit millions" ( $P B$ 25), "légataire", "fortune" ( $P B$ 26), "l'argent de la ville" (A 122)), l'argent étend à l'infini son réseau d'influence et son pouvoir destructeur :

C'était, disons, la nouvelle maison que mes parents avaient enfin achetée avec une partie de l'héritage de ma grand-mère; et en ce sens on peut dire que cette maison était chargée d'histoire, que l'argent qui a servi à l'acheter était lui-même chargé d'histoire, que l'argent en général est chargé d'histoire, mais disons, tout argent qui est passé dans les mains de mes parents l'est encore plus. (PB 57)

Comme on s'en doute, cette histoire qui naît de l'argent renvoie tout naturellement au roman, à cet art supérieur de raconter des histoires, qu'il s'agisse de celui écrit par le personnage ou par Tanguy Viel. Cette interdépendance se donne à lire de manière implicite dans les bagages de Louis lors de ses deux voyages, de Brest à Paris, puis de Paris à Brest. À l'aller, la valise contient l'argent volé à la grand-mère et au retour le manuscrit vengeur. Le double secret caché dans la valise symbolise la métamorphose des billets en mots et de l'argent en roman.

\section{Romans du mensonge}

10 Romans français par le goût des affaires familiales et financières, Paris-Brest et Article 353 sont, on l'a vu, saturés de mensonge en tous genres. Escroquerie, trahison, tromperies en constituent le matériau narratif. La litanie est impressionnante: dans Paris-Brest, le père accusé d'avoir détourné l'argent du club de football quitte la ville "chassé par le mensonge et la calomnie" (PB 39); Louis, avec la complicité du fils Kermeur cambriole sa propre grand-mère; les parents de Louis lui mentent pour s'emparer du manuscrit compromettant. Rien de bien plus glorieux dans Article 353: “'Une vulgaire histoire d'escroquerie', monsieur le juge, rien de plus." (A 17). Cette histoire est symbolisée par un oxymore quasi balzacien : "Ici, bientôt, le Saint-Tropez du Finistère" (A 63). En brouillant la géographie par une mauvaise métaphore, en organisant la rencontre des contraires (la Bretagne et le Sud), la formule renvoie à toutes les impostures. On ajoutera à ce tableau le mensonge de Martial Kermeur qui cache l'achat d'un appartement à Le Goff, et à son propre fils sous prétexte de lui venir en aide (A 353). Et c'est bien la fable du "Corbeau et du renard" qui exemplifie le pouvoir de la flatterie sur la vanité et la cupidité du pauvre monde (A 97).

11 Au mensonge que l'on fait aux autres, s'ajoute le mensonge que l'on se fait à soi-même. La mauvaise foi (au sens sartrien) pose la question de la liberté, du choix et de l'engagement dans la vie. Est-on libre de signer? D'une certaine manière, cette question hante tous les personnages qui s'interrogent ou refusent de s'interroger sur leur responsabilité. "Je n'ai pas le choix" : la phrase revient à plusieurs reprises dans Paris-Brest. Ainsi, à propos d'Albert qui quitte le Cercle marin et va rencontrer la grandmère : “Il est possible de penser qu'il s'était fait croire à lui-même qu'il ne pouvait pas descendre, ou lui avait fait croire à elle qu'il ne pouvait pas descendre, ayant ou n'ayant pas calculé son coup mais attendant qu'on l'aide, ou déjà : qu'elle l'aide" (PB 22). À l'incertitude qui concerne les intentions du vieux monsieur se conjugue l'incertitude que manifeste le narrateur condamné à imaginer une intériorité à laquelle il n'aura jamais accès. Il en va de même pour la grand-mère. Si son choix semble clair dès le départ, le "oui” a besoin d'un peu de ruse pour se faire accepter. La mauvaise foi n'est pas loin, déplacée du côté des motivations : "c'était une forme de sentiment qui lui faisait faire ça, se répétant le stylo à la main : 'mais je l'aime, c'est parce que je l'aime 
que je signe"' (PB 28). Louis prétend, lui aussi, qu'il n'avait pas le choix quand il s'est agi de s'établir chez sa grand-mère ${ }^{7}$ ou d'accepter l'amitié du fils Kermeur.

Le mystère ne porte pas tant sur les motivations qui conduisent les personnages : on comprend assez vite que le ressentiment social et familial les fait agir et explique une bonne partie des escroqueries et des mensonges. Il porte principalement sur la responsabilité, sur la capacité de chacun à devenir la source de ses actes. Les romans de Tanguy Viel ne tranchent pas la question: ils installent une zone incertaine, où la volonté et la subjectivité ne sont jamais niées au profit d'un déterminisme généralisé qui innocenterait tout le monde. Les personnages acceptent bon gré mal gré de rendre compte de leurs actes, ils ne se cachent pas longtemps derrière une mauvaise excuse. Les relations de Lazenec et de Martial Kermeur exemplifient ce jeu du chat et de la souris qui emprunte au vocabulaire de la liberté et de l'aliénation : "Vous savez mener votre barque tout seul [...]. Et en même temps qu'il y avait cette balle rebondissante qui faisait plus de dégâts qu'une pierre dans un lac, je dis bien 'en même temps' il y avait quelque chose en moi qui se gonflait d'orgueil ou je ne sais pas, de souveraineté, quelque chose qui disait, oui, c'est vrai, tu sais mener ta barque - et sans voir que lui, Lazenec, dans mon orgueil, dans ma résistance, dans mon libre-arbitre, bientôt il pourrait s'y vautrer comme dans un canapé en cuir dont il aurait lui-même consolidé les coutures" (A 73). Dans cet univers où la volonté ne sait pas ce qu'elle veut, qui est vraiment coupable? Louis, sa mère, le fils Kermeur ${ }^{8}$ ? Qui est responsable du suicide de Le Goff et de l'achat de Martial ? Leur naïveté ? Lazenec? Tout le monde a sa part du fardeau, chacun des personnages hésite entre hasard et destin ${ }^{9}$. Reste alors à lutter contre sa propre dépersonnalisation, à cesser d'être bête pour devenir homme. Tout est dit dans une formule magnifique, proche des moralistes classiques : "on appelle bêtise les heures d'absence à soi-même" (A 88).

\section{Mensonges du roman}

Le romancier n'est pas le dernier des menteurs. Depuis Aristote, le mot "muthos" désigne à la fois le récit et la fiction. Pour l'auteur de La poétique (en opposition avec Platon, plus méfiant à l'égard de mythes), il n'existe nul antagonisme entre la fiction et la vérité; grâce au muthos, l'écrivain emprunte le détour de l'imagination pour comprendre le monde et la société. Ainsi, Tanguy Viel n'hésite pas à mettre en scène l'artifice de tout récit. L'attitude n'est pas neuve; elle est même devenue un véritable lieu commun depuis La recherche de Proust et Les faux-monnayeurs de Gide. À une époque qui réinstalle la tyrannie du référent, qui fait du réel la source de toute légitimité, Tanguy Viel se plaît à rappeler qu'un roman se construit avec des mots et qu'il se fabrique comme un produit d'artisanat attentif aux attentes de la réception. Mais l'originalité de la démarche créatrice est ailleurs. À mi-chemin de Platon qui se méfie du mythe et d'Aristote qui l'utilise comme un instrument de vérité, le romancier de Paris-Brest et d'Article 353 du code pénal joue de l'ambivalence de la fiction. Tantôt, il met en scène la fiction comme un travestissement de la réalité, tantôt, il la considère comme un discours ajusté au monde.

14 C'est avec ludisme et parfois de façon cryptée que Paris-Brest et Article 353 exhibent les procédés de la création romanesque, assumant pleinement ce mensonge qui consiste à substituer une réalité textuelle à une réalité existentielle. Double ou faux-double du romancier, Louis écrit un roman familial dont il raconte volontiers l'élaboration, entre 
passion et calcul. Clairement mise en abyme, la forme romanesque se manifeste aussi d'une manière plus discrète, grâce aux vertus de la métaphore. La maquette du projet immobilier joue un grand rôle dans Article 353, à la fois dans l'intrigue (elle participe au mensonge) et dans la symbolique du roman (qu'elle représente en miniature) : “Là, cette maquette autour de laquelle on se bousculerait bientôt, tous penchés et admirant le sens du détail, cherchant chacun sa propre maison sur les chemins de plastique, c'était comme un circuit de train électrique dans la vitrine d'un magasin de jouets" ( $A$ 45-46). Et comme devant un bon roman réaliste qui s'attache à reproduire le réel avec une exactitude méthodique, Le Goff s'écrie avec enthousiasme : "Ça fait vrai, hein ?" (A 54). Tromperie dans la fiction, la maquette immobilière installe le mensonge dans la diégèse et dans l'écriture, associant l'imagination de l'écrivain et l'escroquerie de Lazenec, qui tous les deux bâtissent un monde de fiction. En plus de la maquette, le roman entretient un lien métaphorique fort avec l'architecture, cet autre art de la construction. Quand Louis revient à Brest avec son manuscrit dans ses bagages, il découvre la nouvelle maison de ses parents :

mon histoire familiale n'est jamais devenue un livre, pour toutes les raisons que j'aurai sûrement l'occasion d'expliquer, mais seulement un manuscrit que j'avais soigneusement rangé dans ma valise, que maintenant pour la première fois j'allais faire entrer dans la maison. J'ai pensé : c'est comme des poupées russes, maintenant dans la maison familiale il y a l'histoire de la maison familiale. (PB 59)

Cette construction gigogne commande toute la dynamique de Paris-Brest: la vie familiale de Louis se transforme en "roman familial", lui-même inséré dans le roman de Tanguy Viel. D'une certaine manière, le romanesque est partout, prenant le réel en écharpe d'une manière indécidable. Où commence l'événement? Où commence son récit? Que s'est-il réellement passé ? La rencontre d'Albert et de la grand-mère donne lieu à des versions différentes, selon qu'elle émane de la vieille dame ("C'est elle qui raconte ça ainsi" (PB 21)) ou de son petit-fils qui imagine la scène ("C'est moi qui raconte ça ainsi" ( $P B$ 22)) ou qui imagine ce qu'aurait pu imaginer sa mère ("tu aurais raconté une autre version de cette histoire" (PB 33)). À propos du "roman familial" de Louis, Tanguy Viel met en scène le travail de l'écrivain, maintenant l'équilibre entre mimésis et création. Voici comment le fils Kermeur devient personnage, entre exhibition et brouillage des identités :

Sauf que dans mon livre, je lui ai donné un autre nom qui n'a pas d'importance. En tout cas on comprend très vite qu'il ne devait pas être là, dans l'intimité de la famille, que déjà la tension monte avec ma mère, enfin, pas vraiment ma mère mais une femme avec des lunettes noires qui enterre sa vieille mère et qui est quand même ma mère dans le roman, parce que c'est raconté à la première personne, donc par moi en quelque sorte, enfin quelqu'un de proche de moi, disons, assez proche pour que les gens qui me connaissent comprennent que c'est moi. (PB 179)

Louis manifeste ainsi une excellente connaissance des procédés, voire des trucs, qui font les romans à succès : "Dans mon livre, oui, je me suis dit que ce serait mieux comme ça, que Kermeur ait fait de la prison à cause du cambriolage. Ce serait plus romanesque" ( $P B$ 176). Ou encore : "Un roman familial sans enterrement, ai-je pensé en l'écrivant, ce n'est pas un vrai roman familial" (PB 71). Au fond, l'essentiel réside dans l'effet produit ; cette attitude de rhéteur se détourne de la vérité factuelle au profit de la réception, non sans une bonne dose de mauvaise foi quand Louis se cache derrière la tradition pour justifier le règlement de comptes: “j’avais considéré qu'il ne fallait épargner personne, compte tenu de l'esprit général de cette famille et compte tenu 
aussi que c'était un roman, et qu'à ce titre de roman il avait bien fallu que je force un peu le trait" (PB 69).

Mais il y a beaucoup plus troublant. En emboîtant les poupées romanesques (roman de Louis dans le roman de Viel), en posant la vie comme matériau de l'écriture et l'écriture comme réorganisation de la vie, l'auteur pousse son lecteur à s'interroger sur l'expérience personnelle qui suscite la créativité. Dans un jeu assez pervers, en multipliant les reflets, en mettant en scène les relations complexes entre le vécu des personnages, le roman de Louis et son propre roman, Tanguy Viel fait exister sa propre vie en creux, comme nécessairement impliquée par la chaîne des représentations. Le lecteur se trouve ainsi confronté à un chiasme inachevé (A : vie de Louis, B : roman de Louis, $\mathrm{B}$ : roman de Viel, A : vie de Viel), à une structure qui désigne la case manquante et appelle à la combler. Tanguy Viel a-t-il des rapports aussi difficiles avec sa mère ? Ne faut-il pas avoir soi-même des comptes à régler pour inventer des contes aussi cruels? Légitimes et insignifiantes à la fois, ces questions sont en quelque sorte écrites par le texte. Mais l'entreprise ne relève-t-elle pas d'une forme d'ironie supérieure? L'échelonnement mimétique ne produit jamais qu'une réalité indécidable, une fiction sans consistance, née cette fois-ci dans l'imagination du lecteur. En jouant de la proximité du mensonge et de la fiction, en choisissant un personnage qui écrit son "roman familial" entre témoignage et imagination, Tanguy Viel brouille les cartes entre le vrai et le faux, le bien et le mal, le monde et sa représentation. Mais si tout le monde ment à sa manière (auteur et personnages) et prend ses aises avec la réalité, Paris-Brest et Article 353 du code pénal tentent, non sans mal, de redonner une dimension morale à la forme romanesque.

\section{Mentir-juste}

Aux mensonges du monde, l'écrivain répond par les mensonges de la fiction. L'univers que construit Tanguy Viel correspond à une société qui a définitivement fait le deuil de ses illusions et qui vit dans une époque dégrisée de toutes formes de lyrisme. Pour être galvaudé, le mot de "postmoderne" correspond très bien à un siècle qui se détourne des philosophies de l'histoire. Pour les personnages comme pour le romancier, le temps n'est plus vectorisé vers un avenir meilleur, qu'il s'agisse d'une société sans classe issue de la Révolution ou au contraire d'une généralisation de la richesse grâce à l'économie de marché. Il est bien question de la "lutte des classes" (A 144), Martial Kermeur revient sur l'espoir qu'a représenté l'élection de François Mitterrand (A 48), mais le contexte économique est déprimant (fermeture de l'arsenal de Brest) et la belle injonction inspirée de Pasolini ("jeter tout leur corps dans la bataille"10 (PB 119)) s'applique ironiquement à la scène très violente qui oppose la mère et la grand-mère de Louis, comme si la seule combativité qui restait avait déserté toute ambition politique. Dans un monde sans boussole, la tentation du cynisme est grande, loin des vieux clivages politiques ${ }^{11}$. Au départ, un constat désenchanté, assez caractéristique de la société contemporaine: "C'est que dans France, me disait le fils Kermeur, dans France il y a rance" ( $P B$ 19). Mais au lieu d'ouvrir en grand sur le large, le personnage se contente de tirer son épingle du jeu.

Les valeurs morales ne valent pas mieux. Louis en fait la douloureuse expérience dans son enfance. Poussé à voler du chocolat dans un supermarché, il se fait prendre par le directeur qui l'admoneste vertement après que le jeune garçon a avoué son petit 
larcin : "Mais ne jamais dire par sincérité qu'on comptait voler. C'est exactement ce que j'ai fait ce jour-là, par inexpérience j'ai marché dans le piège de la sincérité, j'ai dit en pleurant que j'avouais, que je voulais les voler, que je n'aurais pas dû" (PB 88-89). La leçon est bien comprise : désormais, le mensonge se révèle comme la seule porte de sortie dans un monde qui sent le renfermé. Le même désenchantement se lit en filigrane dans les descriptions de la ville de Brest et les tentatives de reconstruction d'après-guerre. Le narrateur d'Article 353 contemple la ville juste avant son arrestation : “j'ai jeté un dernier regard autour de moi, vers la terre abîmée, la mer en contrebas" ( $A$ 12). À l'insu sans doute du personnage, cette "terre abîmée" n'est pas loin de la "terre gast" médiévale ou même du Waste Land d'Ezra Pound. Pour Louis, le constat est le même devant le spectacle de la ville mal reconstruite, prisonnière de son passé et incapable de penser l'avenir : "Alors à Brest, comme à Lorient, comme à Saint-Nazaire, on n'a rien réinventé du tout, seulement empilé des pierres sur des ruines enfouies" ( $P B$ 9).

20 Paradoxalement, dans ce monde peu attrayant, la loi continue à servir de repère et, dans son sillage, le système démocratique reste une référence. Nul populisme, nul complotisme, nul anarchisme dans ces deux romans qui tentent malgré tout de défendre une attitude morale. "Nul n'est censé ignorer la loi, a dit le juge" (A 171) et quand il rappelle le délit commis par Erwan, son commentaire ne témoigne d'aucune complaisance: "il a quand même fait une grosse connerie" (A 99). Martial Kermeur accepte sa responsabilité et la nécessité de se soumettre à la loi, même si la formulation très métaphorique reste passablement ironique ${ }^{12}$. Tantôt, c'est la justice qui est comparée à un volatile : "Sans doute, j'ai l'âme assez coupable pour ne pas être surpris de voir la loi fondre sur moi comme une buse et déjà planter ses griffes dans mes épaules" (A 10). Tantôt, c'est le personnage lui-même qui plane sur le pauvre monde comme un oiseau : "Elle, la mouette, dans son œil rond sans paupière on aurait dit qu'elle insistait pour faire partie de l'histoire, comme un témoin inflexible qui pourrait se tenir à la barre de tous les tribunaux du monde. Et j'avais juste envie de lui dire que j'irais de moi-même, au tribunal, que je n'avais pas l'intention de me soustraire à la loi" (A 11).

21 Les romans de Tanguy Viel ne renoncent ni au libre-arbitre, ni à la loi ou à la démocratie que celle-ci fonde ; mais le lecteur est confronté à un univers traversé par le doute, où les causalités sont troubles, les responsabilités à interroger, les valeurs souvent indécises et la relativité omniprésente. Les instruments utilisés par Lazenec valent pour tous les personnages: “Je crois bien qu'ils s'appellent l'instinct ou l'intuition ou la ruse" (A 105). Face au mensonge, il convient de se défendre par un autre mensonge, de ruser avec la ruse. Paris-Brest et Article 353 sont sensibles aux injustices, aux inégalités, aux rapports de force qui écrasent le faible sans qu'on sache toujours très bien qui est le fort ou qui est le faible. La littérature comme réparation? L'idée serait à la mode dans la France contemporaine ; mais les deux romans ne répondent qu'en partie à cet idéal de justice. Pour Louis qui s'adresse à son frère, l'écriture romanesque joue un rôle positif : "Mais moi, lui ai-je dit, je ne fais pas ça pour faire du mal. Au contraire, je fais ça pour effacer le mal. Et je me souviens, au moment précis où j'ai dit l'expression 'effacer le mal', d'une pichenette j'ai envoyé mon mégot de cigarette brûler dans la cheminée" ( $P B$ 166). Mais les injustices ne sont pas sûres : on partage la sympathie du juge quand il répare à sa manière la vie cassée des Kermeur père et fils ; on comprend (c'est-à-dire que le roman nous amène à comprendre) le ressentiment de Louis confronté à une mère destructrice et influencé (mais non 
innocenté) par de mauvaises fréquentations ; on comprend que le fils Kermeur ait une revanche à prendre sur la petite société du Tout-Brest; mais, en même temps, la sympathie du lecteur a du mal à suivre quand il s'agit pour Louis de voler sa grandmère, de lui mentir et de se réfugier dans une mauvaise foi de victime.

Le travail de l'écrivain consistera donc à mettre le roman au service d'une justice malgré tout, à écrire non seulement ses personnages, mais pour ses personnages et à créer avec eux une sorte de communauté. Il s'agit, à la fois, pour Tanguy Viel, d'être de la tribu, et d'assumer la responsabilité spécifique d'un écrivain, à la fois complice et en retrait. En témoigne le statut de la narration. Les romanciers peuvent choisir d'utiliser des formes d'écriture présentes dans la vie : lettre, confession, témoignage, mémoire, etc. Dans les romans de Tanguy Viel, le narrateur est un personnage qui raconte son histoire; mais quelle forme choisit-il ? À qui s'adresse-t-il ? Dans Article 353, nous n'avons pas un interrogatoire, mais le récit d'un interrogatoire, qui ne reproduit aucun discours connu et se confond avec le roman de Tanguy Viel, dont le destinataire est, naturellement, le lecteur. Autrement dit, on a le sentiment que l'énonciation de l'auteur et celle du narrateur se confondent. Personne et personnage sont différents ; il ne s'agit pas d'une écriture autobiographique, il ne s'agit pas de rapprocher deux subjectivités, mais deux instances narratives, comme si Tanguy Viel, d'une manière non réaliste, mettait son talent de romancier au service de Martial Kermeur ou de Louis et prolongeait leur voix.

Cette parenté structurale et non psychologique met en place un jeu relationnel qui rapproche ou distingue auteur, narrateur et personnages. Ainsi le romancier et le juge d'Article 353 partagent-ils plusieurs traits importants, même si ces points communs, une fois encore, ne sont jamais exempts d'ironie. L'auteur comme le juge maîtrise le temps et l'espace, pénètre au plus profond des êtres, sonde les reins et les cœurs. Comme le Diable boiteux, l'un et l'autre prennent de la hauteur pour découvrir un monde panoramique : "en tant que juge, on est censé avoir comme une vue panoptique sur les affaires de la ville - pas au début, bien sûr, mais peu à peu, au fil des jours, parce qu'à force d'enquêtes, je n'en sais rien parce que je ne suis pas juge mais j'ai l'impression que c'est comme si on montait en ballon au-dessus des immeubles" (A 44). S'il prend de la hauteur, le romancier sait aussi prendre du recul. Ainsi, Louis, pour pouvoir écrire son roman familial part à Paris, "la ville de l'exil positif" (PB 60-61), pour échapper à la tyrannie du lieu natal et à la déréliction de Palavas-les-Flots.

De l'espace, on passe au temps. Raconter une histoire, qu'il s'agisse de Louis ou de Tanguy Viel, c'est se réapproprier la chronologie par la narration. Dans la vie, les événements marchent inexorablement droit devant eux. C'est ce que rappelle l'épisode de la fête foraine. Martial Kermeur, dans une nacelle de la grande roue, se retrouve à la suite d'une maladresse suspendu dans le vide, risquant une chute mortelle. Heureusement, l'employé du manège actionne la machine à l'envers : “alors j'ai senti la nacelle qui s'arrêtait et puis qui doucement redescendait, le même film dans l'autre sens, oui comme si on remontait le temps, qu'on l'effaçait, et qu'il ne s'était rien passé, que je ne m'étais pas accroché au balcon de fer, qu'Erwan n'avait pas pleuré, qu'on n'était jamais montés sur une grande roue - oh cette sensation, je m'en souviens, depuis, j'aurais aimé qu'elle soit possible encore, sentir, que quelquefois dans nos vies s'enclenche la marche arrière" (A 130). Véritable allégorie, la roue devient une machine à remonter le temps, à "faire machine arrière" (A 142), en opposition avec la réalité qui court devant elle et n'efface jamais rien. Or, ce pouvoir de remonter le temps 
appartient au romancier qui se joue des événements qu'il agence à sa guise. Véritable métaphore du roman, la roue symbolise le privilège de l'auteur et engage sa responsabilité face au monde. Si Martial Kermeur ne peut jamais revenir en arrière, retrouver "une page blanche à effacer à la gomme magique" (A 142), son aventure révèle la liberté de l'écrivain qui est maître de l'intrigue - et même de la gomme tant que le roman n'est pas publié.

Personnages, narrateur et romancier, chacun selon ses compétences use des mots pour témoigner de l'“opacité des choses" (A 116) et opérer quelques percées de lumière. Cette tension vers la lumière, à la fois matérielle et intellectuelle, s'apparente à une opération cathartique. Pour atteindre la "lucidité"13, pour dissiper les brumes matinales, le cheminement est long autant qu'hasardeux. C'est, une fois encore, grâce aux mots que l'opération est possible ${ }^{14}$. Et c'est grâce à la littérature qui montre et démontre à la fois, que s'opère la réconciliation nécessaire du sentiment et de l'idée, de l'émotivité et de la raison ${ }^{15}$. D'une certaine manière, Kermeur et son juge symbolisent les deux étapes essentielles de la catharsis aristotélicienne ${ }^{16}$, l'émotion qui bouleverse le corps et l'intelligence qui apporte ses éclaircissements :

Lui, ce qui l'intéressait, c'était une chose plus mentale, comme une équation mathématique qu'il aurait eu à résoudre ou à formuler. Mais moi aussi, je lui ai dit, moi aussi, j'ai besoin de résoudre l'énigme mais je ne suis pas un cérébral, voilà tout. Et encore, je ne suis pas un impulsif, si vous comptez jour après jour ce que représentent six années de patience, six années à croire qu'à la place d'un champignon mal comestible il pourrait y avoir des baies vitrées qui se refléteraient dans le soleil. (A 107)

Devant Martial Kermeur, le juge de plus en plus touché par l'interrogatoire qu'il conduit accomplit la réunion du cœur et de l'esprit ${ }^{17}$. Que fait-il, sinon prendre le texte du code pénal au mot, respectant à la fois l'esprit et la lettre de la justice ? Face aux mensonges de Lazenec, il faut savoir jouer. Dans les romans de Tanguy Viel, on prend souvent, comme le juge, les mots au pied de la lettre. D'une manière plus anodine, le lecteur est souvent ramené au sens littéral; ainsi, avant d'être un célèbre gâteau consommé le jour de Noël par Louis et sa famille ( $P B$ 15), Paris-Brest désigne un itinéraire dont l'aller et retour en train construit tout le roman. Si Louis se conduit comme un Judas envers sa grand-mère, le mot désigne d'abord très matériellement un œil ménagé dans la porte : “J'ai regardé par le judas tous les jours dix fois par jour " ( $P B$ 99). Quant à la célèbre entreprise des "déménageurs bretons" (PB 45), elle renvoie en l'occurrence à des déménageurs authentiquement bretons qui déménagent la famille de Bretagne pour Palavas-les-Flots.

À l'inverse, le romancier compte aussi sur le sens figuré, comme si le mot cette fois-ci, à l'instar de la mouette, était destiné à voyager, à parcourir le champ de l'imaginaire pour circonscrire une réalité que l'on ne peut explorer que par détours et tâtonnements. Dans un monde qui défie la sagacité et la lucidité, la dynamique métaphorique se donne les moyens - et peut-être l'illusion - de capturer un peu de vérité dans le filet des mots. Le système métaphorique, omniprésent dans les romans de Tanguy Viel, joue sur deux plans à la fois : conformément à la rhétorique, les tropes sont l'expression de l'affect et des émotions; en même temps, ces tropes qui mettent en relation les données du réel font du romancier l'éclaireur du monde qu'il déchiffre.

Voici pour terminer une page d'Article 353, dont le réseau d'images est un des plus denses du livre : 
Et au fond de moi, je me disais : qu'est-ce que tu veux savoir, Lazenec, qu'est-ce que tu crois que je sais et qu'est-ce que ça peut te faire? Et pour un instant, c'était comme si le démon à l'intérieur de lui, j'en avais vu la figure sombre et nue, comme si même elle s'était mise à danser sous mes yeux en toutes ces phrases bien tournées qui faisaient comme une partition. Et je me disais encore : maintenant je suis comme une mauvaise herbe qu'il voudrait arracher, une mauvaise herbe dont il craint qu'elle repousse et repousse infiniment, et c'était comme un bras de fer de silence et de phrases truquées, comme si on déplaçait chacun des pions sur un échiquier. Là, dans le décor un peu gris de la mairie, c'était une sorte de Yalta local, quand en quelques poignées de main tout a l'air de se redessiner, lui se demandant à quel piquet moi aussi, âne ou chèvre ou chien battu, à quel piquet il pourrait encore m'enchaîner, et sans même se rendre compte alors que j'y était déjà, enchaîné, comme le chien de la fable. (A 140)

Le romancier, en quelque sorte, prête sa voix au personnage, parle non pas pour lui, mais avec lui, prolongeant par un fondu-enchaîné de métaphores, l'expression naturelle d'un homme émotif qui transforme le microcosme de son ressentiment en un macrocosme, où se mêlent la nature et la culture, les mots et les choses, les arts et la politique, le jeu et la souffrance, les lieux communs et la singularité d'une aventure. Le déluge de références ouvre la douleur du personnage à la totalité du monde que le romancier restitue sans toujours l'élucider dans un extraordinaire entrelacs de relations et de causalités. Au service des personnages dont ils prolongent la voix, les mots de l'écrivain misent sur la fiction et sur la rhétorique pour ruser avec le mensonge.

\section{NOTES DE FIN}

1. Les deux romans paraissent aux Éditions de Minuit, en 2009 pour Paris-Brest, en 2017 pour Article $353 d u$ code pénal. Les références figurent entre parenthèses, après la citation, selon le code suivant: A (pour Article 353 du code pénal) et $P B$ (pour Paris-Brest), suivis du numéro de page.

2. “Article 353 du code de procédure pénale: la loi ne demande pas compte aux juges des moyens par lesquels ils se sont convaincus, elle ne leur prescrit pas de règles desquelles ils doivent faire particulièrement dépendre la plénitude et la suffisance d'une preuve ; elle leur prescrit de s'interroger euxmêmes dans le silence et le recueillement et de chercher, dans la sincérité de leur conscience, quelle impression ont faite, sur leur raison, les preuves rapportées contre l'accusé, et les moyens de sa défense. La loi ne leur fait que cette seule question, qui renferme toute la mesure de leurs devoirs: Avez-vous une intime conviction?" (A 173-174)

3. "Erwan devant la télévision éteinte. Erwan dans la cuisine à me regarder penser. Erwan derrière la vitrine du banquier. Erwan derrière la porte de sa chambre. Erwan sur les pontons à regarder le gros bateau de Lazenec. Et moi je dis que chaque scène est devenue une image fixe dans son cerveau, au point de faire comme la lame d'un cutter qui a fini par lui déchirer la peau ou non pas la peau mais la chair dessus, tirant sur elle en l'effleurant et à la fin son visage intérieur, il fut comme lacéré." (A 98)

4. "voleur", "bandit", "escroc" (PB 42) 
5. "chez le notaire pour signer les papiers officiels" ( $P B$ 27); “j’ai signé le bail” ( $P B$ 144) ; “des mentions très sérieuses, des 'lu et approuvé' et des 'certifié sur l'honneur"' et des "bon pour accord" (A 91).

6. Roland Barthes évoque "les mots coups de fouet" chez Eschyle (Comment vivre ensemble, Paris, Seuil, 2002, p. 208).

7. “Mais je n'avais pas le choix, lui disais-je, ma mère ne m'a pas laissé le choix, c'était ça ou le Sud, et qu'est-ce que tu aurais fait à ma place, la même chose que moi bien sûr, tout sauf le Sud." (PB 15)

8. Le fils Kermeur est le "déclencheur de toute histoire" (PB 76).

9. "Donc le vendredi, le destin, ou disons, le hasard géographique voulait qu'on fasse un bout de chemin ensemble, le fils Kermeur et moi” ( $P B$ 81) ; "ce jour-là, le hasard ou quelque chose comme ça, a voulu qu'elle, la vieille dame, pousse la haute porte de verre quelques secondes après lui, ayant pris le même menu unique qu'on servait là, et elle lui a proposé son bras" (PB 21).

10. Tanguy Viel réutilise la citation de Pasolini ("Jeter son corps dans la bataille") dans le livret qu'il a écrit pour l'opéra de Philippe Hurel, Les pigeons d'argile, créé au Capitole de Toulouse en 2014.

11. "Écoute-moi bien, il a continué, toi tu es d'une famille de droite, disait-il, et moi je suis d'une famille de gauche, et c'est pour ça qu'on s'entend si bien, parce que toi tu n'as pas envie d'être d'une famille de droite et moi je n'ai pas envie d'être d'une famille de gauche." (PB 105)

12. "je ne sais quelle justice naturelle" (A 163) ; "la justice, j'ai dit au juge, il n'y a que les hommes qui peuvent faire ça" (A 171).

13. "La lucidité ? a dit le juge./Oui c'est ça, exactement ça, j’ai dit, la lucidité. Vous avez toujours le mot qu'il faut." (A 123)

14. "est-ce que le silence, c'est comme l'obscurité ? Un trop bon climat pour les champignons et les mauvaises pensées? Maintenant c'est sûr que je dirais volontiers ça, que les vraies plantes et les fleurs, elles s'épanouissent en plein jour, et qu'il faut parler, oui, il faut parler et faire de la lumière partout, oui, dans toutes les enfances, il ne faut pas laisser la nuit ni l'inquiétude gagner" (A 92). On boit beaucoup dans les romans de Viel : "que quelque chose arrive là par l'alcool" ( $P B$ $155)$; "l'odeur encore flottante de nos conversations sortie du fond des verres" (A 123) ; "avec l'alcool et le vent qui faisaient comme deux serre-livres" (A 133).

15. “encore que je n'aie jamais bien distingué entre une idée et un sentiment”, déclare Martial Kermeur (A 137).

16. La "froideur des lois" contraste avec le juge "si émotif" (A 79).

17. "Et le juge avait l'air de compatir, du moins son visage en empruntait tous les signes." (A 126)

\section{RÉSUMÉS}

Dans un monde sans illusions, dominé par le mensonge et la mauvaise foi, les personnages comme le romancier de Paris Brest et Article 353 du code pénal utilisent toutes les ruses de la fiction pour sauver ce qui peut l'être des valeurs humanistes.

INDEX

Mots-clés : roman, fiction, mensonge, mauvaise foi, ruse 
AUTEUR

CLAUDE COSTE

Université de Cergy-Pontoise 